\title{
Diversité régionale de la prise en compte des zones humides et de leurs dynamiques
}

\section{Magalie Franchomme et Claude Kergomard}

\section{(2) OpenEdition}

1 Journals

\section{Édition électronique}

URL : http://journals.openedition.org/developpementdurable/1754

DOI : 10.4000/developpementdurable.1754

ISSN : 1772-9971

Éditeur

Association DD\&T

\section{Référence électronique}

Magalie Franchomme et Claude Kergomard, « Diversité régionale de la prise en compte des zones humides et de leurs dynamiques », Développement durable et territoires [En ligne], Dossier 6 | 2006, mis en ligne le 10 février 2006, consulté le 03 mai 2019. URL : http://journals.openedition.org/ developpementdurable/1754; DOI : 10.4000/developpementdurable.1754

Ce document a été généré automatiquement le 3 mai 2019.

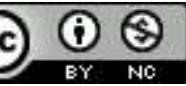

Développement Durable et Territoires est mis à disposition selon les termes de la licence Creative Commons Attribution - Pas d'Utilisation Commerciale 4.0 International. 


\title{
Diversité régionale de la prise en compte des zones humides et de leurs dynamiques
}

\author{
Magalie Franchomme et Claude Kergomard
}

1 Les zones humides souffrent d'une contradiction apparente d'intérêts entre usages et protection des écosystèmes. Mais comme le souligne, R. Mathevet (2004, p. 17) : «la compréhension de leurs valeurs et de leurs fonctionnements s'avère d'une importance capitale si la société veut se donner les moyens de les utiliser durablement et de les conserver». Cela supposerait de tenir compte des difficultés particulières de conservation et de gestion des zones humides, et de la contribution de ces dernières aux politiques de préservation de la diversité biologique, de gestion des ressources en eau et de prévention des inondations.

2 L'affirmation selon laquelle ces zones constitueraient un nouvel enjeu pour l'aménagement du territoire ne doit pas occulter les stratégies contradictoires qui se sont nouées à travers les siècles autour de leur mise en valeur et/ou de leur réduction. Ces contradictions se reflètent dans les pratiques d'aménagement et jusque dans l'évolution des textes législatifs et réglementaires relatifs aux zones humides (1). A titre d'exemple, en région Nord-Pas-de-Calais, caractérisée par une pression anthropique ancienne et importante ayant favorisé souvent l'assèchement des zones humides, leur présence en milieu périurbain constitue une originalité, mais aussi une entrave au développement urbain. C'est pourquoi à travers l'analyse de deux ensembles de zones humides de la région Nord-Pas-de-Calais, la vallée de la Haute Deûle et le val de la Sensée, toutes deux situées, à des degrés divers, en milieu périurbain, nous nous interrogerons sur les modifications dans les pratiques de gestion et de réaménagement des zones humides.

3 Ces questionnements nous conduisent à mener une rétrospective sur la place des zones humides dans les politiques d'aménagement des espaces naturels du Nord-Pas-de-Calais (2) et nous permettent de pointer les disparités dans le processus d'intégration des zones humides de la région (3). 


\section{De fortes disparités spatiales dans leur mode de gestion et leur aménagement}

4 Longtemps considérées comme insalubres et improductives, les zones humides connaissent un regain d'intérêt par la prise en compte de la flore, de la faune et de leurs fonctions. Un grand nombre de mesures éparses à diverses échelles relatives aux zones humides (convention de Ramsar, Loi du l'eau, Directive Cadre Européenne sur l'Eau) constitue, selon nous, autant d'indices d'une reconnaissance progressive et finalement de leur protection.

\subsection{Des aménagements sous-tendus par des impératifs économiques}

Les zones humides ont été, au cours de ces derniers siècles, victimes de leur mauvaise réputation (Fustec et

$\mathrm{Du} \mathrm{XI}^{\mathrm{e}}$ au XIII ${ }^{\mathrm{e}}$ siècle, les zones humides connaissent une première phase de transformations sous forme de création d'étangs, de dérivation des cours d'eau (moulins... ) et de drainage sous l'influence des féodaux et de l'Eglise (Derex, 2001b). L'époque médiévale se caractérise par la généralisation de l'extension des terres cultivées. La seconde phase est concomitante $d u$ renforcement du pouvoir de l'Etat et de la promulgation d'un édit royal au XVII ${ }^{\mathrm{e}}$ siècle visant au dessèchement de tous les marais $\mathrm{du}$ royaume (endiguement et canalisation; De Dienne, 1891). Du XVI ${ }^{\mathrm{e}}$ au XVIII ${ }^{\mathrm{e}}$ siècle, du fait de la situation géopolitique, le Nord de la France est le théâtre d'enjeux militaires qui se traduisent par la construction du "pré-carré » (Dubois et Renard, 1984): les marais viennent en complément défensif des places-fortes et des zones inondables qui s'égrènent en arrière de la ceinture de fer définie par Vauban (Dubois et al., 2000). Dès le XVIII siècle, la volonté de plier la nature aux exigences de l'homme conduit à la conquête de nouveaux espaces considérés par les Physiocrates comme des espaces improductifs et nuisibles (Derex, 2001a). Au XIX ${ }^{\mathrm{e}}$, les sociétés ont mis à contribution leur technicité pour transformer les zones humides et généralement contrôler les niveaux d'eau à leur convenance (Barnaud, 1998). En parallèle, les théories hygiénistes considéraient les eaux stagnantes comme responsables de l'infection de l'air ambiant, des maladies endémiques ou épidémiques. Ainsi, les ingénieurs se sont efforcés de dériver, équiper ou canaliser les fleuves et rivières au détriment des zones humides riveraines. Les marais ont été drainés, asséchés ou comblés pour valoriser l'exploitation agricole ou autoriser l'urbanisation (Billaud, 1984 ; Guillerme, 1997). Cette dégradation lente mais progressive s'est accélérée à la fin des années 1960 du fait de la mise en valeur agricole et du développement de l'agriculture intensive. Le mouvement connait son paroxysme avec la loi d'orientation agricole du 4 juillet 1980 qui définit « la maitrise de l'eau comme facteur essentiel de la production agricole » et encourage par conséquent le drainage à grande échelle (Narcy, 2004). Les surfaces drainées ne cessent d'augmenter jusqu'au début des années $1990^{1}$ (Bernard, 1994; Cizel, 2001). La dégradation des zones humides ne s'est pas opérée au même rythme sur l'ensemble du territoire français et à l'échelle régionale, selon qu'elles 
aient ou non été susceptibles de constituer le support d'activités économiques dynamiques (Carcaud et Garcin, 2001).

\subsection{Des mesures éparses à diverses échelles comme indicateur d'une prise en compte des zones humides}

7 A partir des années 1970, la société remet en cause l'exploitation abusive des ressources naturelles. Les notions de «fonctions écologiques » et de « valeurs » des zones humides se sont répandues au cours des dernières décennies (Fustec et ${ }^{2}$.

Parmi les mesures éparses tendant à une reconnaissance des zones humides, on peut citer la loi sur l'eau du 3 janvier 1992. Elle contribue à la transposition dans le droit français des préoccupations internationales, tout en conférant un statut juridique aux zones humides et des outils pour mettre en œuvre leur préservation. De même, la loi sur l'eau considère qu'elles contribuent à un " patrimoine commun de la nation ». Dans cet esprit, la nécessité d'une "gestion équilibrée » et globale de la ressource est affirmée à l'échelle $\mathrm{du}$ bassin versant. Autrement dit, la loi sur l'eau encourage à la prise en compte de ces espaces. Toutefois, le texte législatif n'est pas assorti «d'obligations précises, ni de compétences de protection clairement énoncées et attribuées » (Romi, 1992, p.14).

Il s'agit également du Programme national de recherche sur les zones humides (PNRZH) dont l'objectif était de mettre en évidence le caractère d' « infrastructures naturelles » des zones humides et la diversité de leur fonctions (hydrologiques, biogéochimiques et biodiversité...). Autant de fonctions justifiant la considération de ces milieux par les différentes politiques qui influent sur leur devenir.

11 C'est aussi, la directive « habitat $~^{456}$, les aides financières à la populiculture et la Taxe sur le Foncier Non Bâti qui incitent à la «valorisation » des terrains en zones humides. La dualité persistante entre la délimitation des espaces naturels et des incitations plus ou moins prononcées en faveur de la transformation des zones humides, aboutit à de fortes disparités spatiales dans leur mode de gestion et leur aménagement.

Cette dualité s'exprime notamment au travers des mesures agro-environnementales instaurées dès 1992 par la PAC qui encouragent à des « méthodes de production conçues pour protéger l'environnement et préserver l'espace rural » et constituent l'un des outils $\mathrm{du}$ développement rural. A ce titre, les pratiques agraires doivent être favorables à l'environnement et conformes au principe de l'éco-conditionnalité (26 juin 2003). Aussi, l'octroi d'aides publiques est assujetti au respect de ces normes.

En écho à ces actions, la Directive Cadre pour une politique communautaire dans le domaine de l'Eau (DCE) renforce l'intérêt fonctionnel des zones humides, qui constituent des milieux d'interface.

Enfin, ce dispositif est renforcé par la récente loi relative au développement des territoires ruraux ${ }^{7}$ dont plusieurs articles concernent la préservation, la restauration et la valorisation des zones humides comme l'exonération de taxe foncière ou régime d'acquisition foncière en zone périurbaine. En zone urbaine, la protection des milieux naturels ne doit plus se faire en confrontation avec le développement économique, mais, conformément aux nouveaux principes du droit de l'urbanisme, entretenir des relations d'équilibre quant à l'usages du sol et la protection de la ressource (Narcy, 2004). 
15 Nul doute que les zones humides sont le résultat d'enjeux stratégiques et socioéconomiques qui ont influencé l'organisation et le développement des territoires (Mermet, 1992). D'ailleurs, la valorisation des zones humides dans l'aménagement peut participer à de vastes opérations de renouvellement urbain, comme nous le démontrerons dans cet article. Finalement, une approche spatialisée de l'usage du sol offre un regard critique sur les aménagements et les techniques d'exploitation de la ressource, mais aussi sur la mise en place de la réglementation et des conditions socioinstitutionnelles du développement des territoires.

2. Le particularisme régional au service d'un aménagement territorial concerté

\subsection{L'originalité des zones humides de la région Nord-Pas-de-Calais}

17 Paradoxalement, eu égard aux caractéristiques topographiques et hydrologiques de la région Nord-Pas-de-Calais, la fraction de la surface des zones humides n'y représente qu'1\%, contre 3\% en moyenne pour la France métropolitaine (SDAGE Artois-Picardie, 1997). Les roselières, tourbières alcalines, étangs et marais, prairies humides et mares prairiales constituent une mosaïque de milieux (IFEN, 2004). La grande originalité des zones humides du Nord-Pas-de-Calais tient à l'anthropisation ancienne de ces paysages, aboutissant à une fragmentation des espaces naturels. Les rivières sont marquées par d'importants aménagements hydrauliques qui ont artificialisé les principaux cours d'eau. Les zones humides sont encore souvent victimes des pratiques agricoles intensives (drainage, populiculture) et de l'artificialisation du sol (urbanisation et industrialisation), d'autant plus qu'une grande part des zones humides (environ $80 \%$ ) n'est ni incluse dans le réseau Natura 2000, ni dans les périmètres de protection ${ }^{8}$ (arrêté de protection de biotope, réserves naturelles volontaires ...).Nous nous intéressons particulièrement aux zones humides de petite taille, résiduelles et non incluses dans un dispositif de protection nationale ou internationale. Nos deux terrains d'étude : le val de la Sensée et la vallée de la Haute Deûle, sont reconnus en tant qu'espaces «naturels» (inclus dans des ZNIEFF), mais ne sont pas engagés dans un dispositif de protection, tels que les parcs régionaux ou les réserves volontaires (figure 1). 


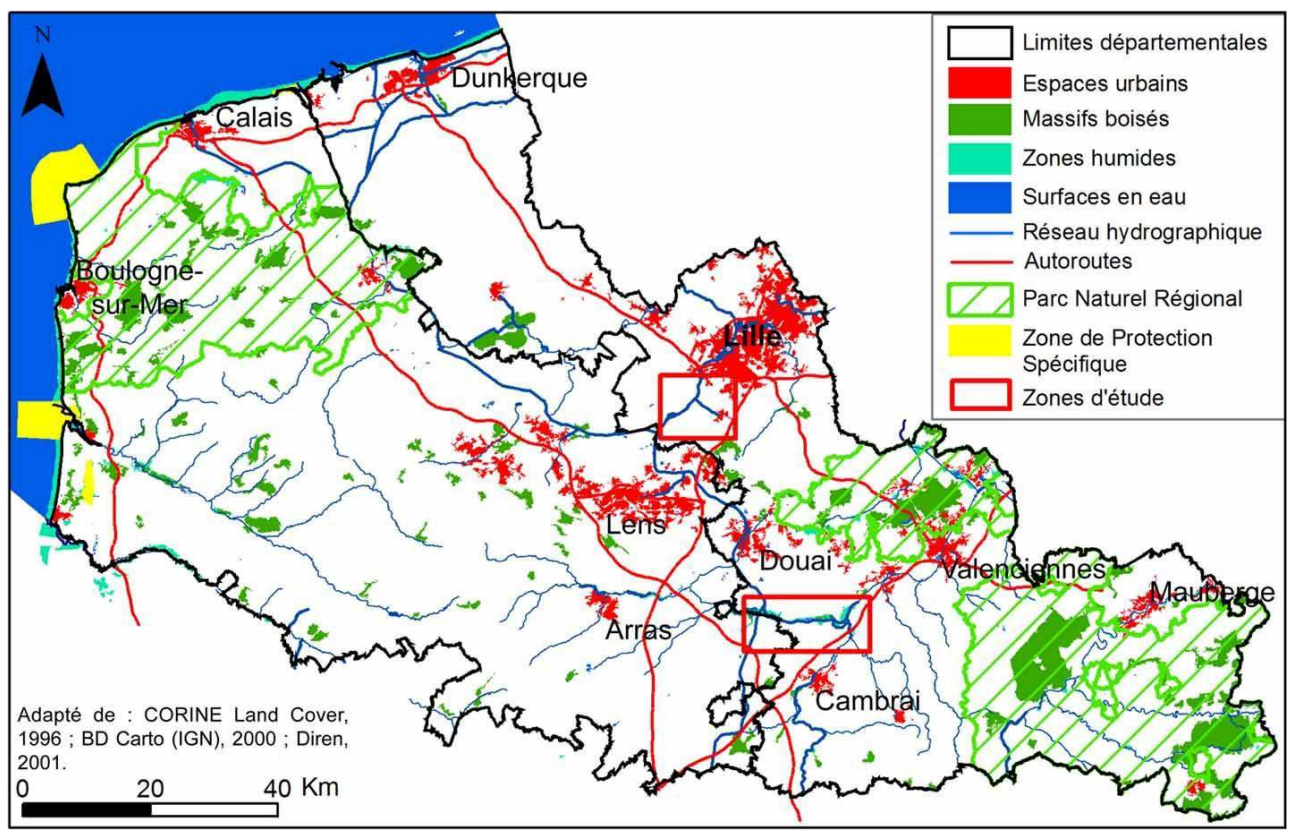

Les compétences récentes confiées au conseil régional dans le domaine de l'environnement, favorisent les démarches intégrant les spécificités locales dans une logique régionale et européenne de maintien de la biodiversité et de restauration des espaces naturels.

\subsection{Une ambition régionale : le dispositif environnemental régional en faveur de la conservation des espaces naturels?}

Dans un contexte particulier marqué par le morcellement des espaces naturels, la région Nord-Pas-de-Calais s'est dotée d'une stratégie relative à la mise en place d'une «trame verte ». La trame verte a pour vocation de créer une base de concertation à partir d'un dispositif régional environnemental, dont l'objectif est le maintien d'espaces dédiés à la biodiversité. Cette réflexion est développée autour des notions de "cœurs de nature " (ou «noyau de biodiversité ») remarquables par la présence d'espèces faunistiques et floristiques exceptionnels et de « corridors écologiques » devant favoriser la biodiversité aux échelles régionale et européenne. Ces derniers ne constituent pas obligatoirement une continuité physique entre les cœurs de nature, il peut s'agir de corridors étapes ou de corridors potentiels. Cette méthodologie permet d'envisager tout d'abord des continuités éco-paysagères, basées sur des critères géomorphologiques, d'occupation du sol, et d'intérêt faunistique et floristique, et des espaces à "renaturer » ou à conforter. Néanmoins, la trame verte régionale n'est pas systématiquement compatible avec les aménagements réalisés à l'échelle locale et n'est actuellement qu'en cours de réalisation. Elle se fonde sur la segmentation de la région Nord-Pas-de-Calais en unités paysagères homogènes qui ne concordent pas systématiquement avec les échelons décisionnels. Ainsi, la continuité éco-paysagère entre la métropole lilloise et le bassin minier tend à se confronter à des difficultés inhérentes à la structuration territoriale. Les moyens de mise en œuvre sont, en ce qui concerne le volet foncier, le recours au droit de préemption et la 
contractualisation, notamment à partir des ZNIEFF et des Espaces Sensibles définis par le département. Les zones humides jouent un rôle prépondérant dans la trame verte.

\section{Des disparités régionales dans la gestion des zones humides}

La vallée de la Haute Deûle et le val de la Sensée répondent aux objectifs d'intégration régionale, avec toutefois la prise en compte des spécificités du milieu et du dynamisme des acteurs locaux.

\subsection{Les zones humides périurbaines : espace de réinvention}

21 Le territoire de Lille-Métropole se caractérise par un déficit en espaces publics dédiés à la nature et aux loisirs par rapport à des métropoles de taille et d'influence comparable (15 $\mathrm{m}^{2} /$ hab. pour l'arrondissement de Lille, contre $70 \mathrm{~m}^{2} / \mathrm{hab}$. à Cologne). En effet, on ne recensait, en 1993, que 515 ha d'espaces verts et 298 ha d'espaces boisés ouverts au public dans la Communauté Urbaine de Lille. L'importante fréquentation des espaces verts suffit à démontrer le manque d'équipements récréatifs de niveau métropolitain (LMCU, 2002). Par ailleurs, les espaces verts urbains apparaissent très dispersés et non, ou mal, reliés entre eux.

Le sud-ouest de la métropole lilloise se distingue notamment par l'importance des ressources en eau et la présence de champs captants irremplaçables qui exploitent la nappe aquifère de la Craie et qui satisfont $35 \%$ des besoins en eau de l'agglomération. Ces derniers sont extrêmement menacés par les risques de pollution agricole et industrielle et par l'intensification de l'urbanisation. La pérennisation des stations de pompage et l'amélioration de la qualité des eaux superficielles et souterraines sont affirmées par l'institution d'un Projet d'Intérêt Général. Ce double enjeu a conduit à l'inscription, dans le Schéma Directeur, de 10000 hectares d'espaces verts d'ici à 2015 par l'intermédiaire de la création d'une trame verte métropolitaine (figure 2). 
Figure 2 : La trame verte dans le cadre du Schéma directeur de Lille-Métropole

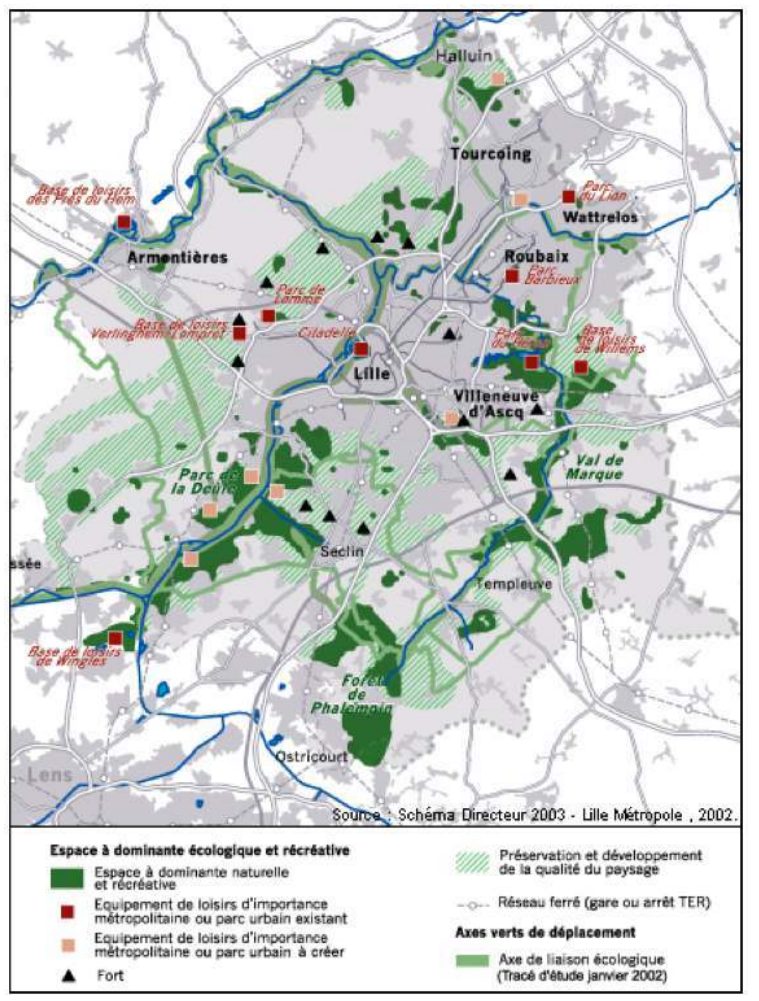

Source : http://www.lille-metropole-2015.org/schemdir/index.htm

Les rives de la Haute Deûle ont été retenues pour le projet du fait de l'existence de territoires propices au développement d'espaces de nature et de loisirs (marais, prairies humides, espaces boisés, friches industrielles) et de la densité des champs captants (figure 3). Un syndicat mixte d'aménagement a été constitué le 9 décembre 1993 pour mener la réalisation. Ce syndicat regroupe, depuis 1999, les communes de HouplinAncoisne, Santes, Wavrin, Don, Seclin, Haubourdin et Lille Métropole Communauté Urbaine, multipliant ainsi les acteurs. Il peut être considéré comme la première étape marquante de la mise en œuvre d'une coopération intercommunale pour l'aménagement d'un grand espace naturel métropolitain. Le parc de la Deûle, inauguré dans le cadre des festivités de Lille 2004, est un projet remontant au premier Schéma Directeur métropolitain de 1968. Il devait former à l'origine un axe vert reliant l'agglomération lilloise aux communes du bassin minier. L'aménagement d'espaces à vocation récréative est concomitant d'une nouvelle politique d'aménagement en rupture avec l'histoire des deux derniers siècles de la vallée. 
Figure 3 : Les zones humides et le parc de la Deûle dans le cadre de la trame verte métropolitaine

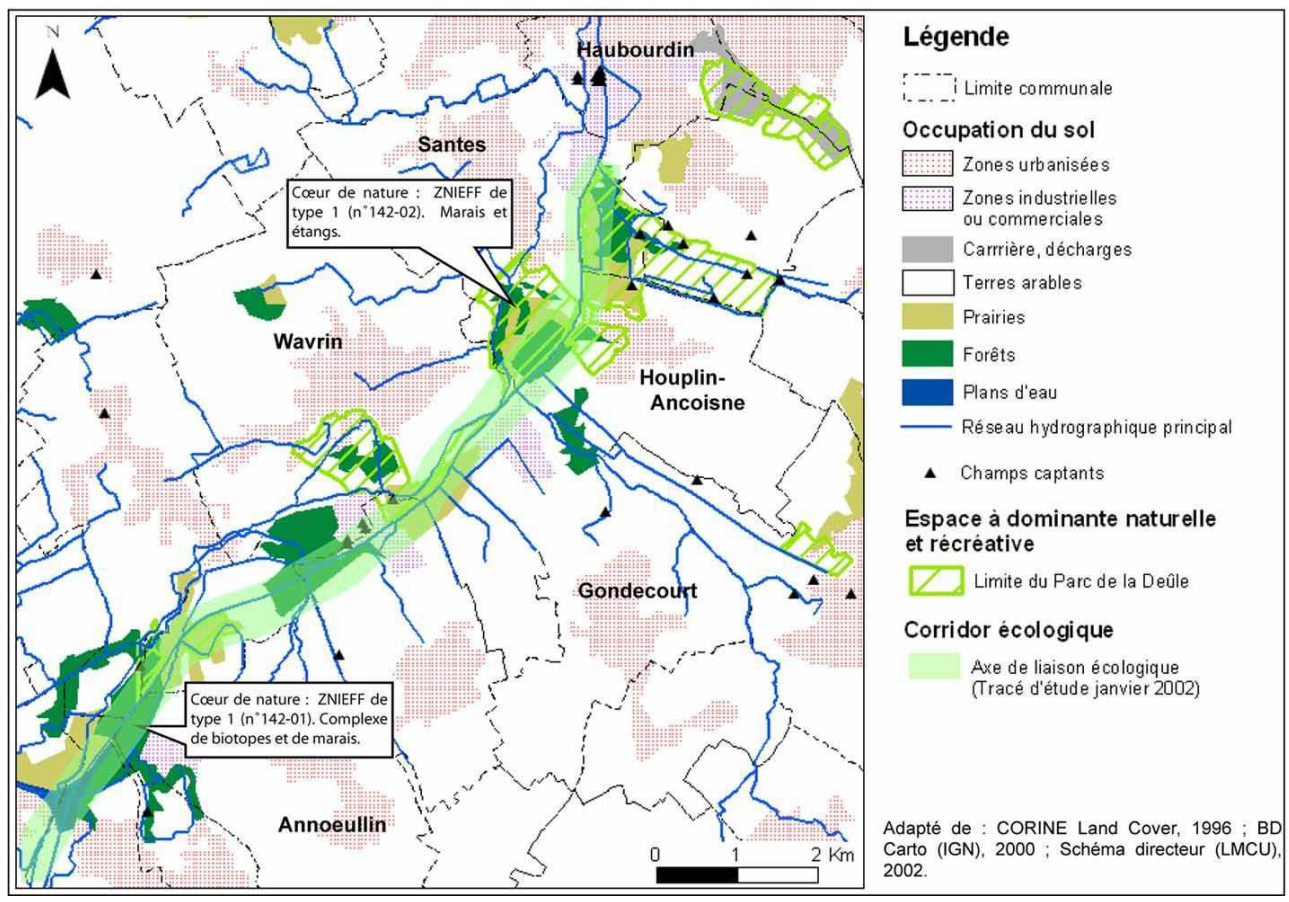

Les espaces humides constituent l'un des axes fédérateurs du parc, même si ces zones « à fort potentiel naturel» ne concernent qu'une proportion limitée du territoire (LMCU, 2002). La vallée de la Haute Deûle est passée du statut d'espace agricole en périphérie de Lille à celui d'espace périurbain. L'étalement urbain, l'accès à la propriété et à la maison individuelle se sont ajoutés aux pressions agricoles et industrielles. La canalisation du cours d'eau (inaugurée au XIII ${ }^{\mathrm{e}}$ siècle et élargie en 1970) et le drainage des marais ont conduit à la disparition de ceux-ci (Dessaux et al., 1998). Il subsiste à leur emplacement un couloir de friches industrielles, de cultures, de peupleraies, de bosquets marécageux et de petits étangs présentant un intérêt faunistique moyen (hivernage de rapaces et de quelques oiseaux d'eau). Paradoxalement, malgré ce potentiel écologique relatif, ces espaces se voient actuellement préservés par la prise en compte de leur multifonctionnalité à la fois sur le plan hydrologique (biodiversité, rétention des polluants ...) et sur le plan de l'aménagement territorial. En effet, le fait que ces zones humides soient situées au milieu d'une région très urbanisée augmente fortement leur intérêt écologique. A ce titre, elles sont inscrites dans une stratégie globale relative à la mise en œuvre de la trame verte (sous la désignation de « cœur de nature »).

Dans ce contexte, les travaux d'aménagement du parc ont intégré un espace récréatif, de découverte de la nature et des activités agricoles; des équipements culturels et scientifiques, sous forme de postes d'observation ont été créés. Une intégration paysagère des installations a été envisagée en privilégiant les matériaux naturels, notamment les structures en bois, minimisant l'impact visuel. Une réflexion redonnant une cohérence à un paysage morcelé a également été menée. Le projet tend ainsi à limiter la consommation foncière urbaine et ses effets induits tout en favorisant le renouvellement urbain. L'ensemble se conjugue pour aboutir à une multifonctionnalité des usages propres aux dynamiques locales et aux spécificités du milieu. Cependant, ce schéma d'intégration n'est pas transposable à l'intégralité des zones humides de la région. 


\subsection{Le val de la Sensée : une absence de vision commune}

Située entre la Scarpe et de l'Escaut, la vallée marécageuse de la Sensée (affluent de l'Escaut) s'étire d'Ouest en Est sur une trentaine de kilomètres (figure 4). Large d'un kilomètre, le val de la Sensée sépare le Douaisis périurbain au Nord, du Bas Artois rural au Sud, multipliant les entités administratives auxquelles il se rapporte. La vallée se compose, en effet, d'une trentaine de communes, réparties sur les deux départements du Nord et du Pas-de-Calais, ainsi que sur quatre arrondissements. Il en résulte un maillage administratif complexe, peu fonctionnel en termes de gestion et d'aménagement du territoire.

Figure 4 : Un patrimoine naturel dominé par les zones humides : le val de Sensée

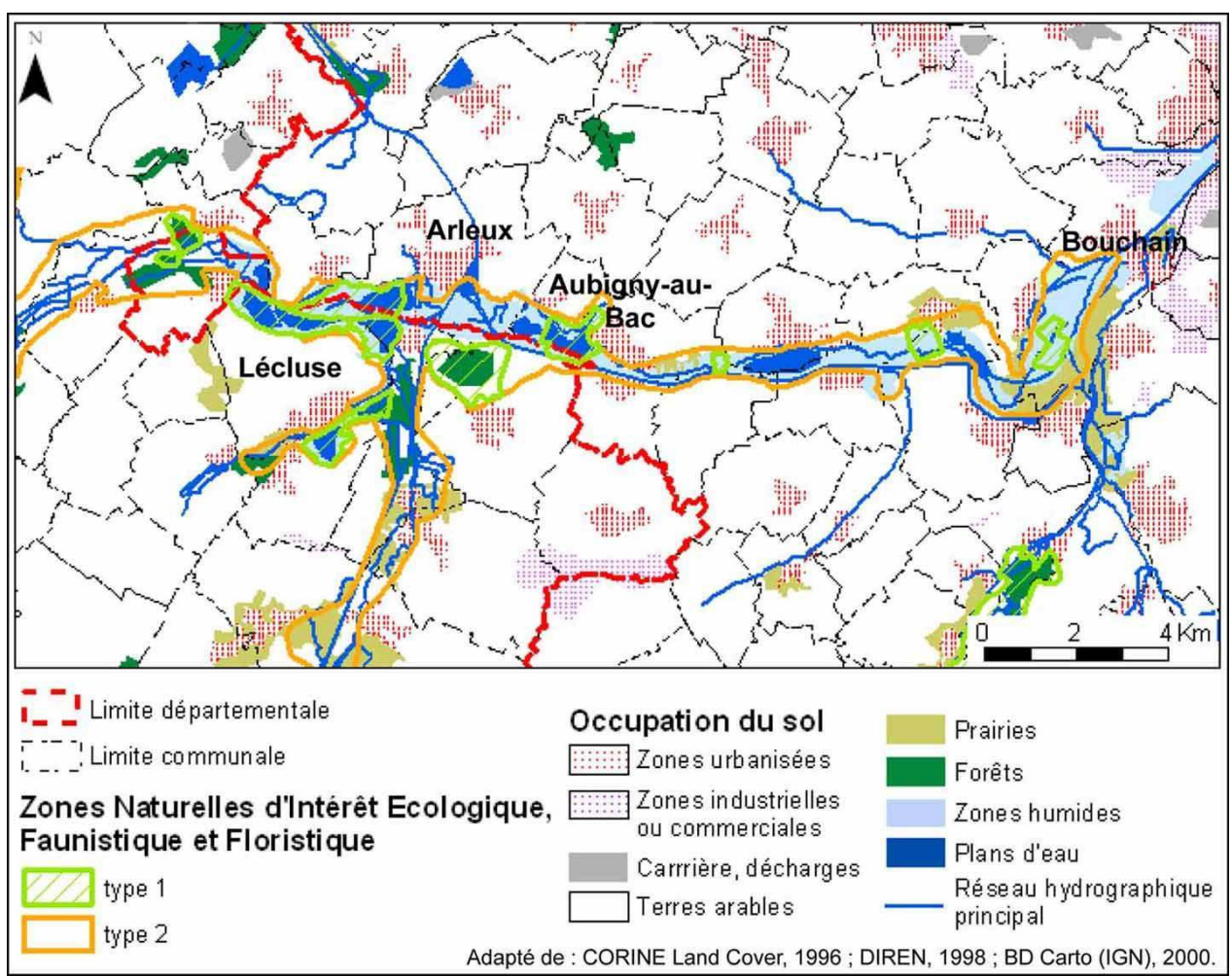

L'influence des conditions naturelles a été accentuée ou atténuée par les travaux

d'aménagement effectués depuis dix siècles (Lecocq, 1998). À partir du VII ${ }^{\mathrm{e}}$ siècle, le cours d'eau a été durablement façonné par l'homme (détournement vers les étangs, travaux de creusement de canaux de navigation: canal de la Sensée, canal de l'Escaut et Canal du Nord), perturbant le réseau hydrographique (Hydratec, 2004). La rivière de la Sensée est aujourd'hui scindée en deux parties: la Sensée amont (mince filet qui se jette dans le Canal du Nord) et la Sensée aval. L'affleurement de la nappe de la Craie et la faible pente de la vallée conditionnent l'existence d'étangs et de marais, de canaux de drainage ou de navigation, de peupleraies et d'espaces agricoles. Selon les fiches ZNIEEF de la DIREN, les zones humides couvrent environ $3000 \mathrm{ha}$, dont 800 ha de plans d'eau. L'influence ancienne de l'homme associée à la dynamique naturelle de la végétation s'est traduite par une grande diversité de biotopes conférant à ce complexe tourbeux une valeur paysagère et une richesse biologique de premier ordre et expliquant l'inscription d'une grande 
partie de la vallée en ZNIEFF 1 (dont les sites n¹2-02, 12-05, 12-09), en ZNIEFF 2 (site n¹2) et sous la désignation de « noyau de biodiversité ».

La pression humaine est également intense du fait des nombreuses activités de loisirs qu'offre le secteur : chasse, pêche, tourisme. La chasse (322 huttes en 2003) et la pêche (environ 4500 pêcheurs) sont des activités fort valorisées. Ces usagers (pêcheurs et chasseurs) s'approprient et aménagent le milieu. Mais l'intensification des interventions, notamment dans les marais de chasse, entraîne une artificialisation du fonctionnement des zones humides. L'artificialisation du milieu est accentuée par les activités touristiques. Le tourisme est ancien, puisqu'il émerge en 1936 (lors des premiers congés payés) avec la création d'un premier parc de loisirs (la "plage») dans la commune d'Aubigny-au-Bac. Il a connu une affluence exceptionnelle durant les années 1950 et 1960 (environ 200000 visiteurs par an, Dewailly, 1967), concernant alors la population ouvrière du Bassin Minier proche. Même si le lieu n'est plus aussi fréquenté, il a conservé un pouvoir attractif. L'attrait du val de Sensée réside dans la présence des plans d'eau et des activités annexes : camping, canotage, voile, ski nautique... Les constructions diverses, composées de matériaux disparates (planches, tôles,...) sont implantées de façon anarchique et souvent sans autorisation. Elles entraînent le mitage de la vallée, le tout ceinturant étangs et cours d'eau privatisés résultant de l'importante spéculation foncière, compromettant la valeur touristique du site et l'équilibre écologique de la vallée.

\section{Conclusion}

31 L'étude de zones humides résiduelles et non incluses dans un dispositif de protection nationale ou internationale a permis de mettre en exergue de fortes disparités dans l'évolution des zones humides de la région Nord-Pas-de-Calais. Ces différences tiennent moins à des caractéristiques physiques du milieu qu'à leur situation géographique et à leur passé. L'intégration des zones humides de la vallée de la Haute Deûle et du val de la Sensée dans l'aménagement du territoire répond à des enjeux spécifiques; les politiques d'aménagement du territoire dépendent fortement de la nature de ces enjeux. La préservation, la restauration et la valorisation des zones humides ne s'opèrent donc pas au même rythme. Face à la nécessité de préservation de la ressource en eau et du milieu naturel en zone périurbaine, la mise en valeur des zones humides des rives de la Haute Deûle s'est orchestrée autour d'une politique de création d'espaces verts. La création du parc de la Deûle affirme, dans le cadre d'une politique d'aménagement, le caractère multifonctionnel des espaces naturels. A l'inverse, le val de la Sensée se singularise par une multifonctionnalité de fait, ancienne et qui semble vouée à perdurer. Dans les deux cas cependant, la gestion dans le cadre d'usages multiples et générateurs de conflits reste à inscrire dans une politique du développement durable régional. 


\section{BIBLIOGRAPHIE}

Barnaud G., 1998,

Bernard P., 1994, Les zones humides, rapport d'évaluation, Comité interministériel de l'évaluation des politiques publiques, Premier Ministre - Commissariat Général du Plan, rapport d'évaluation, La Documentation française, $391 \mathrm{p}$.

Billaud J-P., 1984, Marais Poitevin. Rencontres de la terre et de l'eau, L'Harmattan, Paris, 265 p.

Carcaud N., Garcin M. (coord.), 2001, Géoarchéologie de la Loire moyenne et de ses marges. Synthèse des résultats du PCR 1996-1999, $125 \mathrm{p}$.

Cizel O., 2001, «Etat actuel de la réglementation sur les zones humides en France », Premières rencontres nationales du pôle-relais zones humides intérieures, Parc naturel régional de la Forêt d'Orient, 29-30 novembre 2001, Parcs nationaux régionaux de France, 19 p.

De Dienne, 1891, Histoire du dessèchement des lacs et des marais du royaume avant 1789. Paris, Champion et Guillaumin, $570 \mathrm{p}$.

Derex J-M., 2001a, La gestion de l'eau et des zones humides en Brie (fin de l'Ancien Régime - fin du XIXème siècle), Paris, L'Harmattan, 551 p.

Derex J-M., 2001b, « Pour une histoire des zones humides en France (XVII ${ }^{\mathrm{e}}$-XIX ${ }^{\mathrm{e}}$ siècle). Des paysages oubliés, une histoire à écrire ", Histoire et Sociétés Rurales, $\mathrm{n}^{\circ} 15,1^{\mathrm{er}}$ semestre 2001, pp. 11-36.

Dessaux N, Révillon S, Delchambre S., 1998, "Stratégie d'exploitation et mutations du paysage dans la vallée de la haute Deûle (Nord) : l'exemple du marais de Seclin du $13^{\mathrm{e}}$ au $19^{\mathrm{e}}$ siècle ", Revue du Nord, Tome LXXX, n³26-327, pp. 531-571.

Dewailly J-M., 1967, « Utilisation du sol et aménagement d'une vallée humide : La vallée de la Sensée ", Hommes et Terres du Nord, n¹, pp. 60-74.

Donadieu P., 1996, Paysages de marais. Ed. Jean-Pierre De Monza, Paris, 199 p.

Dubois J-J., Renard J-P., 1984, « Forêts et frontières : quelques réflexions pour une étude causale et évolutive ", Espaces, Populations, Sociétés, n¹, pp. 25-42.

Dubois J-J., Kergomard C., Laganier R., « Analyse géohistorique des paysages d'eau de la région de Condé-sur-l'Escaut (Nord) », Hommes et Terres du Nord, n², 2000, pp. 77-86.

Fustec E., Lefeuvre J-C. et coll., 2000, Fonctions et valeurs des zones humides, Dunod, Industries Techniques, série Environnement, 425 p.

Guillerme A., 1997, Les temps de l'eau - La cité, l'eau et les techniques (fin III ${ }^{e}$-début XIX ${ }^{e}$ siècle), Seyssel, Champs Vallon, $186 \mathrm{p}$.

Hydratec, 2004, Etude hydraulique globale dans le cadre du SAGE de la Sensée, Hydratec, 36 p.

IFEN, 2004, L'environnement en Nord-Pas-de-Calais, IFEN, DIREN Nord-Pas-de-Calais, 180 p.

Johannès J.M. (Ed.), Huglo C., De Malafosse J. (dir.), 1998, Juris-classeur - Environnement, Editions du Juris-Classeur, Collection des Juris-Classeurs, vol. 2. 
Lecocq C., 1998, Quand l'Escaut était affluent de la Satis, de la Satis à la Scarpe en passant par la Sensée et l'Escaut : une affaire de détournement majeur, Association SATIS, 25 p.

Lefeuvre J-C., Dame R.F., 1994, « Comparative studies of salt marshes processes in the New and Old World : an introduction ", In: Mitsch W.J. (Ed.), Global wetlands. Old World and New, Ed. Elsevier, Amsterdam, pp. 169-179.

Le Louarn P. (Ed.), 1999, Les zones humides et le droit. CNFPT, SFDE, Lille, 270 p.

LMCU, 2002, Schéma directeur de développement et d'urbanisme de Lille métropole, Agence de développement et d'urbanisme de Lille Métropole, $254 \mathrm{p}$.

Mathevet R., Mauchamp A., Grillas P., 2002, « Multi-usage et conservation des zones humides ou quel développement durable pour la Camargue? », Faire Savoir n², pp. 33-39.

Mathevet R., 2004, Camargue incertaine. Sciences, usages et natures, Buchet/Chastel, Meta-Editions, Paris, 206 p.

Narcy J.B., 2004, Pour une gestion spatiale de l'eau. Comment sortir du tuyau ?, P.I.E. Peter Lang, Coll. Ed. EcoPolis, 343 p.

Romi R., 1992 : Les espaces humides. Le droit entre protection et exploitation des territoires, L'Harmattan, Dossier Environnement, Paris, 122 p.

Scarwell H., Laganier R., 2002, «Eau et intercommunalité dans la Région Nord-Pas-de-Calais : état d'avancement des SAGE à travers l'exemple du SAGE de la Canche », BAGF-Géographies, n¹, pp. 104-123.

SDAGE Artois-Picardie, 1997, L'état des Lieux, SDAGE Artois-Picardie, Vol. 2, 59 p.

\section{RÉSUMÉS}

Le constat de la dégradation et de la régression spatiale des zones humides ont conduit à partir des années 1970 à dresser des typologies, mener des inventaires et engager des actions de préservation. Cependant, les zones humides de faibles superficies, souvent relictuelles, se situent aujourd'hui en dehors des dispositifs de protection. Pourtant, en raison de leurs multiples fonctions, elles sont progressivement intégrées aux politiques d'aménagement du territoire. Les zones humides constituent ainsi un nouvel enjeu de développement. Elles sont l'objet de stratégies contradictoires quant à leur valorisation. L'étude de deux exemples situés en région Nord-Pas-de-Calais permet de s'interroger sur la reconnaissance et l'intégration de la multifonctionnalité de ces espaces dans les politiques d'aménagement.

The fact of the wetlands decline and spatial recession since 1970 are leading to draw up classifications, inventories and to start preservation actions. Indeed, small areas wetlands are generally kept apart from most studies and protection policies as well. Nevertheless, the local and regional planning gradually integrates them, because small areas wetlands have different hydrological and biological functions. The wetlands constitute a new development contribution, despite contradictions between their protection and their uses. Two examples in the Nord-Pasde-Calais Region (France) show the evolution of the practices and review different ways of integration of the wetlands in regional planning. 
INDEX

Mots-clés : aménagement du territoire, multifonctionnalité, Nord-Pas de Calais, zones humides Keywords : different ways, regional planning, wetlands

\section{AUTEURS}

\section{MAGALIE FRANCHOMME}

Allocataire-monitrice d'Enseignement Supérieur en géographie à l'Université de Lille 1, spécialisée dans le domaine de gestion des zones humides, a coordonné avec Helga Scarwell, le colloque «Contraintes environnementales et gouvernance des territoires » (Editions de l'Aube nord), magalie.franchomme@ed.univ-lille1.fr, Tél. 03.20.33.60.68.

\section{CLAUDE KERGOMARD}

Professeur de géographie à l'Ecole normale supérieure, est spécialisé dans le domaine des interactions environnement et société. Il a coordonné le projet de recherche « Gouvernance et territoires face à l'émergence des stratégies du développement durable : les espaces à forte contrainte environnementale de la région Nord-Pas-de-Calais » soutenu par le PEVS du CNRS. claude.kergomard@ens.fr 